new method which is easy, take little time and brought a good result exprimentally and clinically, we reported its technique and results.

Technique: 1) We catch a pilot tread on the end, and scarred tissues at the end is resected to the part where the nerve fibers surely extend. 2) On the other hand, a silicone tube is cut longitudinally and the four threads in total are caught on the two ends. The nerves are sutured with atraumatic needle with 0OS2 nylon thread. 3) Next, the silicone tube covered on the one end of the nerve is introduced by the pilot thread, so that the nerve is inserted in the lengh of a half of the tube, and fixed on the epineurium with the two threads caught on previously. 4) The same manipulationis done for the other end and the resected section should be fixed in surplus to attach. 5) The pilot thread is removed and the suture finishes.

Experimental Methods and Their Results: We used tibial nerves of adult :abbits, then we cut them off and sutured by our new method. In 3 weeks after operation, no scar was observed near sutured line, and its histological findings reveal the throng of the fibers facting the peripheral side from the central portion. In E.M.G. findings, only fibrillation voltage was observed in 3-4 weeks after operation, but we recognized low amplitude voltage after 8-11 weeks. In 26 weeks after operation almost normal discharge is shown.

Clinical Result: As we got the experimental results of our method of nerve suture, as is mentioned above, we clinically applied it to the 7 cases of injured nerves.

The nerves sutured already by our method are 5 cases of median nerves and a case of ulnar nerve at the wrist, and a case of radial nerve at the upper site of the elbow. In all cases, we got satisfactory results clinically and electromyographically.

\title{
61. Experimental Study on the Hemodynamics of Spinal Cord (Part 3)
}

\author{
Kengo Tsumekawa, Naohiro Ohgushi and Masanao Ikeda \\ Department of Surgery, Kyoto Univ. School of Medicine
}

Our experimental study were designed to make the ischemia of thoracic segments of spinal cord with ligation of bilateral intercostal arteries in dogs.

Changes of oxygen tension at the thoracic segment of spinal cord and changes of skin temperature at forelimb and hindlimb were measured in the 8-th. laminectomized dogs and used as our experimental indicators.

Speciments of spinal cord after sacrificing the dog were also histologically 

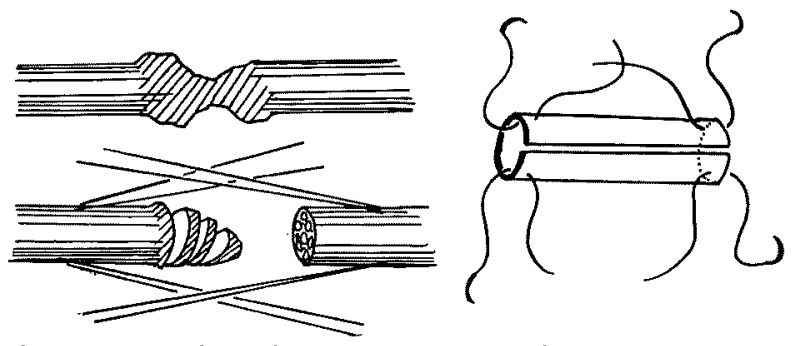

Fig. 1. Resection of neurom \& Preparation of Silicon-tube.

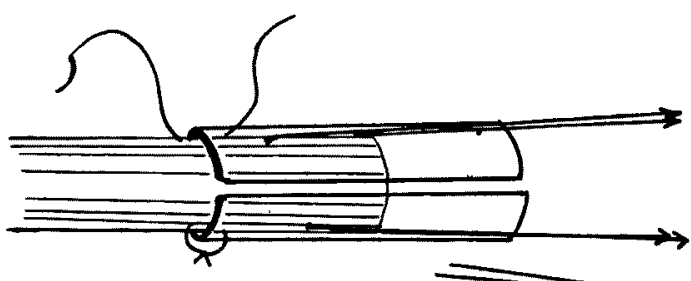

Fig. 2. Insertion \& Fixation (a).
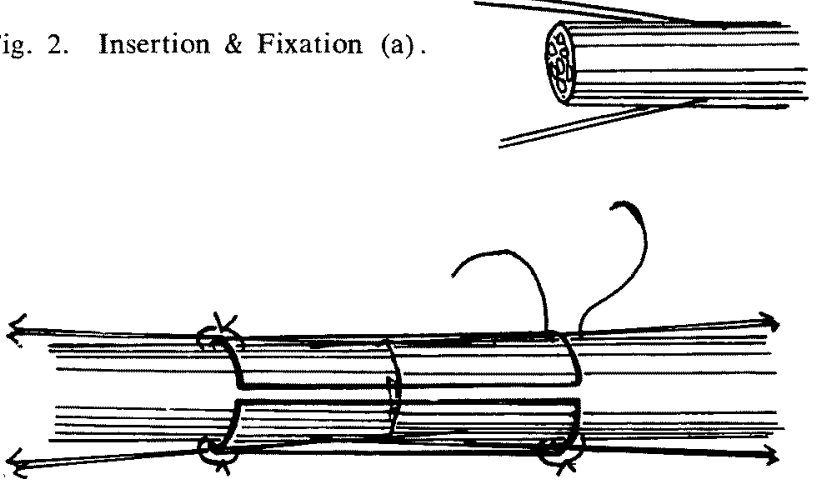

Fig. 3. Insertion \& Fixation (b).

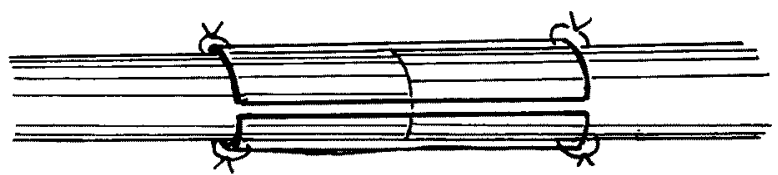

Fig. 4. Finish.

examined with hematoxylin-eosin and cresyl violet stain. For histological examination speciments of not-laminetomized dogs were used as control.

As our experimental results it was confirmed that the oxygen tensions after the ligation of bilateral three to five intercostal arteries were not decreased below $10 \mathrm{~mm}$. $\mathrm{Hg}$.

After the ligation of bilateral seven to nine intercostal arteries, they decreased gradually untill below $10 \mathrm{~mm}$. $\mathrm{Hg}$ within $30-60 \mathrm{~min}$. after the surgery.

After the ligation of bilateral seven to nine inter-costal arteries, difference 
of skin temperature between forelimb and hindlimb was occured thirty minutes after the surgegry. Hindlimb is warmer as forelimb.

The histological changes of spinal cord after the ligation of bilateral three to five intercostal arteries wer not observed in all specimens of spinal cord, which were sacrificed and excised at the period of each 30,60 and $90 \mathrm{~min}$. after the surggery.

The histological changes after the ligation of bilateral seven to nine intercostal arteries were already occured and slightly observed in the speciments, which were sacrificed at the time of $30 \mathrm{~min}$. after the surgery.

This changes became histologically significant in the specimens at the period of $60 \mathrm{~min}$. and $90 \mathrm{~min}$.

Observation of chronic experiments.

Spastic paraplegia, motor weakness, incontinentia urinae and alvi, hyperreflexia were observed in two cases within five cases after the ligation of bilateral nine intercostal arteries. These phenomenon were not observed in another groups.

The treatment of spinal cord ischemia are now in progress by our clinic.

\title{
62. Circulatory Dynamies of the Spinal Cord; An Experimental Study Using ${ }^{32}$ P-labeled Erythrocytes
}

\author{
Fumio Kato \\ Department of Orthopaedic Surgery, University of Tokyo
}

To investigate the dynamic aspect of circulation of the canine spinal cord, ${ }^{32} \mathrm{P}$-labeled erythrocytes were injected intraarterially and its circulation curve was recorded on the dorsal surface of the fourth cervical cord. The recording system consisted of a surgical end-window G-M tube, a rate meter and a recorder.

Of 34 experiments, 20 were considered to be suitable for analysis. In normal circulation curves, the average appearance time was $5.0 \mathrm{sec}$. (SD $1.4 \mathrm{sec}$.), and the average time from apprearance to peak was $9.3 \mathrm{sec}$. (SD $2.7 \mathrm{sec}$.). The peak amplitude of radioactivity was variable. Occlusion of unilateral vertebral artery or both common carotid arteries caused no remarkable change of the circulation curve. When both vertebral arteries were occluded, the appearance time was delayed to $19.8 \mathrm{sec}$. in average. It was as late as $43.3 \mathrm{sec}$. when both vertebral and carotid arteries were occluded.

The results suggest the significance of the ample collateral circulation to the cervical spinal cord, although the direction of flow of the individual vessels remains to be studied. 